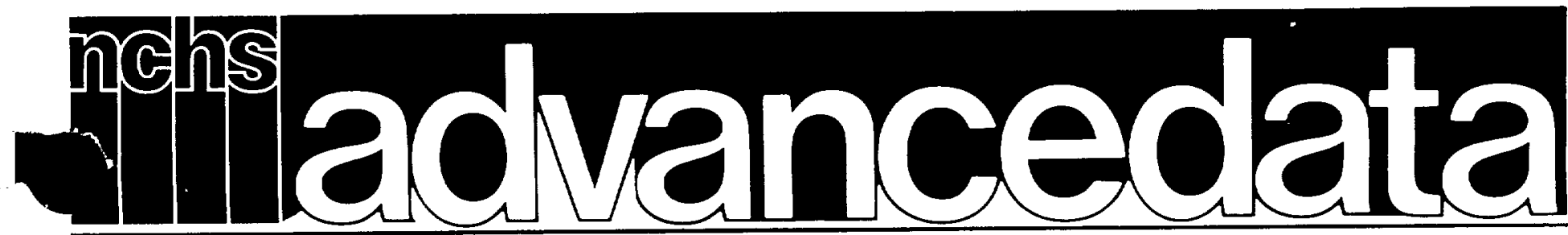

From Vital and Health Statistics of the National Center for Health Statistics

January $18,1985 \bullet$ Number 105

\title{
Diagnosis-Related Groups Using Data From the National Hospital Discharge Survey: United States, 1982
}

\author{
by Robert Pokras and Kurt K. Kubishke, Division of Health Care Statistics
}

\section{Introduction}

This report presents selected estimates for 1982 of diagnosis-related groups (DRG) based on data from the $\mathrm{Na}$ tional Hospital Discharge Survey (NHDS). A similar report has been published for 1981.1 The current plan is to publish ports on DRG's regularly because they determine the reimrsement rates of Medicare inpatients, about 30 percent of all discharges from short-stay non-Federal hospitals. ${ }^{2}$

Developed at the Yale School of Organization and Management, DRG's are being used by the Health Care Financing Administration, some States, and some third party payers to reimburse hospitals for inpatient care on a prospective basis. ${ }^{3}$ This approach to health care reimbursement operates on the principle that patients with similar medical conditions should receive similar care and use approximately the same amount of resources; therefore, in general a hospital should be reimbursed the same amount for each patient in a DRG. While there is variation in resource consumption among individuals within a DRG, these are expected to balance across all patients.

DRG's were developed under the guiding principle that "The primary objective in the construction of DRG's was a definition of case type, each of which could be expected to receive similar outputs or services from a hospital."4 Their formulation was accomplished using clinical judgment and statistical procedures that classify patients by measuring resource utilization. The first step in this process was to cluster the universe of medical diagnoses into broad, mutually exclusive categories. These groups were formed to be consistent in their anatomical or physiopathological classification, or in a manner in which they are clinically managed. Once these vajor diagnostic groups were formed, an interactive statistical gram (AUTOGROUP ${ }^{4}$ ) was used to further classify each Aajor group into discrete DRG's. This process incorporated patient information regarding diagnoses (primary and secondary), procedures, sex, and age to explain maximally a patient's length of stay. In all, there currently are 470 DRG's.

Prospective reimbursement was authorized under the Tax Equity and Fiscal Responsibility Act of 1983. Under this act hospitals participating in the Medicare program were brought into this system beginning with their fiscal year as of October 1, 1983. The Health Care Financing Administration, which operates the Medicare program, is allowing several years for hospitals to make a transition to prospective reimbursement by adjusting DRG payments based on certain hospital characteristics and geographic location. At the end of this phase-in period, care provided to a Medicare inpatient will translate into a preestablished payment based solely on the patient's DRG.

There is an important issue related to the NHDS and the implementation of this prospective reimbursement system: how this system may affect the selection of a patient's principal diagnosis and/or comorbidities. Because the NHDS is designed to collect data on the morbidity of the hospital inpatient population, any external influence on diagnostic practices may affect NHDS data. For example, two patients admitted to a hospital for treatment of chest pain-one diagnosed as having chest pain and the other diagnosed as having angina-will be placed into different DRG's and have different reimbursement rates. There is speculation that in cases such as this prospective reimbursement may influence the selection of a diagnosis, ${ }^{3}$ which in turn may affect estimates produced from the NHDS. After prospective payment has been in place for a few years, it may be possible to examine trends in NHDS data and determine the magnitude, if any, of this type of effect.

The statistics in this report are based on data collected by means of the NHDS, a continuous survey conducted by the National Center for Health Statistics since 1965. Data for this survey are sampled from short-stay non-Federal general and specialty hospitals in the 50 States and the District of Columbia. The sample for 1982 contained approximately 214,000 medical 
records from 426 hospitals. The relevant variables required to produce DRG's (diagnoses, procedures, sex, age, and other variables) were abstracted from the face sheet of each sampled medical record, and NHDS data thereby could be used to produce national estimates of DRG's. These estimates may be of value for hospitals to compare their experience with that of other hospitals. For this reason, statistics in this report are frequency estimates and associated average length of stay for DRG's by hospital bed size and region of the country.

\section{Highlights}

The frequency and average length of stay for the most common DRG's are presented by age, region of the country, and hospital bed size (tables 1-4). Age is dichotomized as under 65 years of age and 65 years of age and older. This allows a comparison with the Medicare population because Medicare covers most hospital costs for approximately 94 percent of discharges 65 years of age and over. Tables 1 and provide regional data, while tables 3 and 4 provide bed-st data for these DRG's. Within each of these sets of tables, the first (tables 1 and 3 ) are for patients under 65 years of age, and the second (tables 2 and 4 ) are for patients 65 years of age and over.

By definition, some DRG's are only for patients in a specific age range. In such a case the DRG title and the table title (tables 1-4) together define the age group of the estimate. That is, the most restrictive case of either the table or DRG title determines the age group of the estimate. For example, "Diabetes, age greater than 35 years" in table 2 only refers to patients 65 years of age and over because of the table title; whereas, "Simple pneumonia and pleurisy, age greater than 69 years

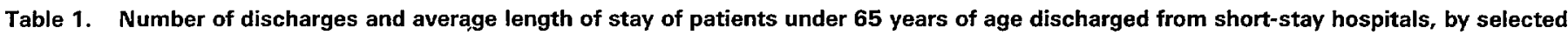
diagnosis-related groups and geographic regions: United States, 1982

[Discharges from non-Federal short-stay hospitals. Excludes newborn infants]

\begin{tabular}{|c|c|c|c|c|c|c|c|c|c|c|}
\hline Diagnosis-related group & $\begin{array}{l}\text { All } \\
\text { regions }\end{array}$ & Northeast & $\begin{array}{l}\text { North } \\
\text { Central }\end{array}$ & South & West & $\begin{array}{l}\text { All } \\
\text { regions }\end{array}$ & Northeast & $\begin{array}{l}\text { North } \\
\text { Central }\end{array}$ & South & West \\
\hline & \multicolumn{5}{|c|}{ Number in thousands } & \multicolumn{5}{|c|}{ Average length of stay in days } \\
\hline All discharges $\ldots \ldots \ldots \ldots \ldots \ldots \ldots \ldots$ & 27,896 & 5,564 & 7,929 & 9,804 & 4,598 & 5.9 & 6.5 & 6.3 & 5.6 & 5.0 \\
\hline $\begin{array}{l}\text { Vaginal delivery without complicating } \\
\text { diagnoses } \ldots \ldots \ldots \ldots \ldots \ldots \ldots \ldots\end{array}$ & 2,784 & 524 & 765 & 937 & 559 & 2.9 & 3.4 & 3.3 & 2.7 & 2.2 \\
\hline $\begin{array}{l}\text { Medical back problems } \ldots \ldots \ldots \ldots \ldots \\
\text { Esophagitis, gastroenteritis, and } \\
\text { miscellaneous digestive disorders, } \\
\text { age } 18-69 \text { years without substantial }\end{array}$ & 790 & 121 & 245 & 298 & 125 & 7.2 & 9.0 & 7.3 & 6.8 & \\
\hline $\begin{array}{l}\text { comorbidity and/or complication. ....... } \\
\text { Cesarean section without substantial }\end{array}$ & 673 & 97 & 178 & 320 & 78 & 4.1 & 4.5 & 4.2 & 4.2 & 3.3 \\
\hline $\begin{array}{l}\text { comorbidity and/or complication. ....... } \\
\text { Nonradical hysterectomy, age less than } \\
70 \text { years without substantial comorbidity }\end{array}$ & 649 & 125 & 145 & 258 & 121 & 5.7 & 6.7 & 6.2 & 5.3 & 4.8 \\
\hline 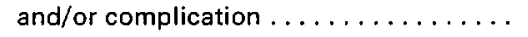 & 495 & 70 & 128 & 212 & 85 & 7.2 & 7.9 & 7.6 & 7.1 & 6.2 \\
\hline $\begin{array}{l}\text { Unrelated operating room procedures.... } \\
\text { Esophagitis, gastroenteritis, and } \\
\text { miscellaneous digestive disorders, }\end{array}$ & 401 & 75 & 116 & 145 & 65 & 11.2 & 13.8 & 12.0 & 9.7 & 10.1 \\
\hline age $0-17$ years $\ldots \ldots \ldots \ldots \ldots$ & 392 & 69 & 111 & 173 & 39 & 3.8 & 3.9 & 3.8 & 3.8 & 3.3 \\
\hline $\begin{array}{l}\text { Psychoses . . . . . . . . . . . . . . . } \\
\text { Alcohol- and substance-induced organic }\end{array}$ & 388 & 95 & 118 & 108 & 68 & 16.5 & 19.1 & 19.7 & 13.8 & 11.6 \\
\hline $\begin{array}{l}\text { mental syndrome } \ldots \ldots \ldots \ldots \ldots \ldots \ldots \\
\text { Dilation and curettage of uterus; }\end{array}$ & 360 & 157 & 90 & 68 & 45 & 10.6 & 9.8 & 11.9 & 10.7 & 10.9 \\
\hline $\begin{array}{l}\text { conization except for malignancy ....... } \\
\text { Abortion with dilation and curettage }\end{array}$ & 345 & 111 & 85 & 118 & 32 & 1.8 & 1.7 & 1.9 & 2.0 & 1.2 \\
\hline of uterus. . . . . . . . . . . . . & 325 & 136 & 65 & 86 & 39 & 1.3 & 1.0 & 1.7 & 1.5 & 1.1 \\
\hline $\begin{array}{l}\text { Bronchitis and asthma, age } 0-17 \text { years... } \\
\text { Simple pneumonia and pleurisy, age }\end{array}$ & 313 & 65 & 96 & 118 & 34 & 3.9 & 4.6 & 3.8 & 3.8 & 3.3 \\
\hline $\begin{array}{l}0-17 \text { years } \ldots \ldots \ldots \ldots \ldots \ldots \\
\text { Tonsillectomy and/or adenoidectomy, }\end{array}$ & 279 & 36 & 86 & 131 & 28 & 4.7 & 5.3 & 5.0 & 4.6 & 4.1 \\
\hline $\begin{array}{l}\text { age } 0-17 \text { years } \ldots \ldots \ldots \ldots \ldots \ldots \ldots \\
\text { Inguinal and femoral hernia procedures, } \\
\text { age } 18-69 \text { years without substantial }\end{array}$ & 279 & 43 & 106 & 83 & 48 & 1.8 & 1.7 & 1.9 & 2.1 & 1.2 \\
\hline comorbidity and/or complication. ...... & 271 & 76 & 74 & 80 & 42 & 4.2 & 4.0 & 4.4 & 4.7 & 3.0 \\
\hline $\begin{array}{l}\text { Diabetes, age greater than } 35 \text { years } \ldots . . \\
\text { Vaginal delivery with sterilization } \\
\text { and/or dilation and curettage of }\end{array}$ & 259 & 52 & 72 & 104 & 30 & 8.2 & 9.9 & 8.7 & 7.4 & 6.4 \\
\hline uterus. . . . . . . . . . . . & 247 & 44 & 57 & 110 & 37 & 3.6 & 4.2 & 4.1 & 3.3 & 2.8 \\
\hline $\begin{array}{l}\text { Other factors influencing health status... } \\
\text { Total cholecystectomy with common } \\
\text { bile duct exploration, age less than } \\
70 \text { years without substantial comorbidity }\end{array}$ & 242 & 53 & 68 & 75 & 46 & 3.5 & 3.6 & 3.8 & 3.2 & 3.4 \\
\hline $\begin{array}{l}\text { and/or complication } \ldots \ldots \ldots \ldots \ldots \ldots \\
\text { Bronchitis and asthma, age } 18-69 \text { years } \\
\text { without substantial comorbidity }\end{array}$ & 233 & 48 & 63 & 83 & 39 & 7.8 & 8.1 & 7.9 & 8.1 & \\
\hline and/or complication . . . . . . . . . . & 227 & 45 & 62 & 81 & 38 & 5.3 & 5.9 & 5.8 & 4.9 & 4.6 \\
\hline
\end{tabular}


Table 2. Number of discharges and average length of stay of patients 65 years of age and over discharged from short-stay hospitals, by selected diagnosis-related groups and geographic regions: United States, 1982

charges from non-Federal short-stay hospitals. Excludes newborn infants]

\begin{tabular}{|c|c|c|c|c|c|c|c|c|c|}
\hline $\begin{array}{c}\text { All } \\
\text { regions }\end{array}$ & Northeast & $\begin{array}{l}\text { North } \\
\text { Central }\end{array}$ & South & West & $\begin{array}{c}\text { AlI } \\
\text { regions }\end{array}$ & Northeast & $\begin{array}{l}\text { North } \\
\text { Central }\end{array}$ & South & West \\
\hline \multicolumn{5}{|c|}{ Numbers in thousands } & \multicolumn{5}{|c|}{ Average length of stay in days } \\
\hline 10.697 & 2,283 & 3,008 & 3,631 & 1,774 & 10.1 & 12.3 & 10.3 & 9.4 & 8.2 \\
\hline 429 & 81 & 127 & 119 & 102 & 2.9 & 3.0 & 3.3 & 3.0 & 2.4 \\
\hline 427 & 99 & 109 & 157 & 62 & 8.7 & 10.9 & 9.0 & 8.2 & 6.3 \\
\hline 387 & 88 & 108 & 132 & 59 & 9.7 & 11.8 & 10.3 & 8.8 & 7.7 \\
\hline
\end{tabular}

All discharges . . . . . . . . . . .

Lens procedures . . . . . . . . . . . .

Atherosclerosis, age greater than

69 years and/or substantial comorbidity

and complication . ..............

Heart failure and shock. ...........

Esophagitis, gastroenteritis, and

miscellaneous digestive disorders,

age greater than 69 years and/or

substantial comorbidity and

complication .................

Chronic obstructive pulmonary disease...

Specific cerebrovascular disorders

except transient ischemic attack......

Simple pneumonia and pleurisy, age

greater than 69 years and/or substantial

comorbidity and complication .........

Unrelated operating room procedures. ...

Diabetes, age greater than 35 years ....

Angina pectoris................

Medical back problems .............

Cardiac arrhythmia and conduction

disorders, age greater than 69 years

and/or substantial comorbidity

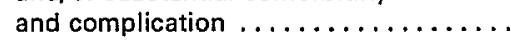

Circulatory disorders with acute

vyocardial infarction without

rdiovascular complications,

scharged alive $\ldots \ldots \ldots \ldots \ldots \ldots \ldots$

Hypertension. ................

Transient ischemic attacks. .........

Bronchitis and asthma, age greater than

69 years and/or substantial comorbidity

and complication . . . . . . . . . . .

Transurethral prostatectomy, age greater

than 69 years and/or substantial

comorbidity and complication ........

Kidney and urinary tract infections,

age greater than 69 years and/or

substantial comorbidity and

complication ................

Respiratory neoplasms . . . . . . . . . .

Hip and femur procedures except

major joint, age greater than 69 years

and/or substantial comorbidity and

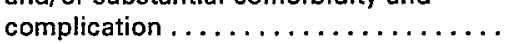

\begin{tabular}{|c|c|c|c|c|c|c|c|c|c|}
\hline 376 & 63 & 102 & 169 & 42 & 6.7 & 8.3 & 6.9 & 6.3 & 5.8 \\
\hline 300 & 54 & 76 & 117 & 54 & 9.8 & 11.9 & 10.2 & 8.8 & 9.2 \\
\hline 295 & 64 & 76 & 103 & 52 & 15.4 & 20.4 & 16.2 & 13.2 & 12.2 \\
\hline 276 & 51 & 80 & 106 & 38 & 10.7 & 11.8 & 10.6 & 10.7 & 9.5 \\
\hline 226 & 59 & 65 & 72 & 29 & 17.3 & 20.0 & 17.5 & 15.8 & 15.0 \\
\hline 208 & 49 & 56 & 77 & 24 & 10.0 & 13.9 & 9.7 & 8.4 & 8.1 \\
\hline 195 & 49 & 47 & 67 & 32 & 6.6 & 8.0 & 6.4 & 6.6 & 4.9 \\
\hline 186 & 38 & 58 & 63 & 27 & 9.3 & 11.5 & 9.7 & 7.7 & 8.8 \\
\hline 181 & 35 & 51 & 65 & 30 & 7.1 & 9.1 & 7.2 & 7.1 & 4.8 \\
\hline 174 & 44 & 44 & 62 & 24 & 12.2 & 13.6 & 12.9 & 11.8 & 9.4 \\
\hline 158 & 28 & 43 & 70 & 17 & 7.9 & 8.8 & 7.7 & 7.6 & 8.4 \\
\hline 155 & 39 & 42 & 52 & 22 & 7.6 & 9.6 & 7.8 & 7.3 & 4.3 \\
\hline 148 & 22 & 38 & 59 & 29 & 8.3 & 9.2 & 8.0 & 8.3 & 8.0 \\
\hline 144 & 29 & 42 & 45 & 28 & 9.3 & 11.1 & 9.5 & 9.4 & 6.8 \\
\hline 142 & 23 & 34 & 67 & 17 & 8.5 & 9.9 & 8.8 & 8.1 & 7.1 \\
\hline 137 & 36 & 34 & 43 & 24 & 10.9 & 11.0 & 11.9 & 11.2 & 9.0 \\
\hline 135 & 27 & 45 & 38 & 25 & 19.5 & 25.1 & 19.9 & 18.5 & 14.3 \\
\hline
\end{tabular}

and/or substantial comorbidity and complication" in table 2 would not include a patient under 70 years of age because of the restriction in the DRG title.

The most common DRG for patients under 65 years of age was "Vaginal delivery without complicating diagnoses" (table 1), with an estimated 2.8 million discharges in 1982. "Cesarean section without substantial comorbidity and/or complication," with 649,000 discharges, also was among the most frequent DRG's in this age group. For patients 65 years age and older (table 2), "Lens procedures" was the most ommon DRG, 429,000, and "Atherosclerosis, age greater than 69 years and/or substantial comorbidity and complication," 427,000 , was the second most common DRG for the elderly.

The average length of stay for specific DRG's in the four regions of the country generally reflects the pattern found for all patients: the Northeast and North Central have the longest average length of stay and the West has the shortest. Regional length-of-stay differences were greater for patients 65 years of age or more than for younger patients. The West had an average length of stay of 5.0 days for patients under 65 years of age, and the Northeast had an average length of stay of 6.5 days, a difference of 1.5 days, or 30 percent greater. For older patients, however, the Northeast had an average length of stay 4.1 days greater than the elderly patients in the West (12.3 versus 8.2 days), a difference of $\mathbf{5 0}$ percent.

Overall there was a tendency for length of stay to increase with hospital bed size (table 3 and 4) for patients under 65 years of age as well as for older patients, but this pattern is not 


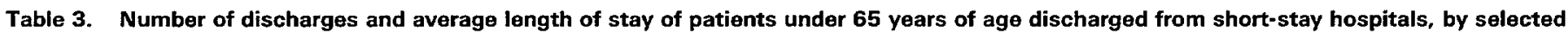
diagnosis-related groups and hospital bed size: United States, 1982

[Discharges from non-Federal short-stay hospitals. Excludes newborn infants]

\begin{tabular}{|c|c|c|c|c|c|c|c|c|c|c|c|c|}
\hline Diagnosis-related group & $\begin{array}{c}\text { A/l } \\
\text { hospita/s }\end{array}$ & $\begin{array}{l}6-99 \\
b e d s\end{array}$ & $\begin{array}{l}100- \\
199 \\
\text { beds }\end{array}$ & $\begin{array}{l}200- \\
299 \\
\text { beds }\end{array}$ & $\begin{array}{l}300- \\
399 \\
\text { beds }\end{array}$ & $\begin{array}{l}500 \\
\text { or } \\
\text { more } \\
\text { beds }\end{array}$ & $\begin{array}{c}\text { All } \\
\text { hospitals }\end{array}$ & $\begin{array}{l}6-99 \\
b e d s\end{array}$ & $\begin{array}{l}700- \\
199 \\
\text { beds }\end{array}$ & $\begin{array}{l}200- \\
299 \\
\text { beds }\end{array}$ & $\begin{array}{l}300- \\
399 \\
\text { beds }\end{array}$ & $\begin{array}{l}500 \\
\text { or } \\
\text { more } \\
\text { beds }\end{array}$ \\
\hline & \multicolumn{6}{|c|}{ Number in thousands } & \multicolumn{6}{|c|}{ Average length of stay in days } \\
\hline All discharges . . . . . . . . . & 27,896 & 4,664 & 4,906 & 4,459 & 6,909 & 6,958 & 5.9 & 4.5 & 5.3 & 5.7 & 6.2 & 6.8 \\
\hline $\begin{array}{l}\text { Vaginal delivery without } \\
\text { complicating diagnoses . . . . . . }\end{array}$ & 2,784 & 425 & 466 & 433 & 700 & 759 & 2.9 & 2.4 & 2.8 & 2.8 & 3.1 & 3.2 \\
\hline $\begin{array}{l}\text { Medical back problems . . . . . . } \\
\text { Esophagitis, gastroenteritis, and } \\
\text { miscellaneous digestive } \\
\text { disorders, age } 18-69 \text { years } \\
\text { without substantial comorbidity }\end{array}$ & 790 & 170 & 137 & 150 & 185 & 149 & 7.2 & 6.3 & 7.0 & 7.5 & 7.6 & 7.6 \\
\hline $\begin{array}{l}\text { and/or complication ......... } \\
\text { Cesarean section without } \\
\text { substantial comorbidity and/or }\end{array}$ & 673 & 191 & 116 & 115 & 143 & 108 & 4.1 & 3.3 & 4.3 & 4.6 & 4.2 & 4.8 \\
\hline $\begin{array}{l}\text { complication . . . . . . . . . . . } \\
\text { Nonradical hysterectomy, age } \\
\text { less than } 70 \text { years without } \\
\text { substantial comorbidity and/or }\end{array}$ & 649 & 75 & 123 & 93 & 175 & 183 & 5.7 & 5.4 & 5.2 & 5.5 & 5.8 & 6.1 \\
\hline $\begin{array}{l}\text { complication . . . . . . . . . . . } \\
\text { Unrelated operating room }\end{array}$ & 495 & 51 & 111 & 80 & 126 & 125 & 7.2 & 7.1 & 6.5 & 7.4 & 7.3 & 7.5 \\
\hline $\begin{array}{l}\text { procedures . . . . . . . . . . . } \\
\text { Esophagitis, gastroenteritis, and } \\
\text { miscellaneous digestive }\end{array}$ & 401 & 50 & 65 & 65 & 98 & 123 & 11.2 & 6.9 & 7.7 & 12.8 & 13.0 & 12.5 \\
\hline disorders, age $0-17 \ldots \ldots \ldots$ & 392 & 82 & 97 & 60 & 78 & 75 & 3.8 & 2.7 & 3.7 & 3.8 & 4.0 & 4.8 \\
\hline $\begin{array}{l}\text { Psychoses . . . . . . . . . . . . } \\
\text { Alcohol- and substance-induced }\end{array}$ & 388 & 34 & 74 & 40 & 137 & 103 & 16.5 & 12.0 & 14.5 & 15.1 & 15.8 & 20.9 \\
\hline $\begin{array}{l}\text { organic mental syndrome. . . . } \\
\text { Dilation and curettage of uterus; } \\
\text { conization except for }\end{array}$ & 360 & 105 & 59 & 39 & 97 & 60 & 10.6 & 9.6 & 13.5 & 12.9 & 9.9 & 9.4 \\
\hline $\begin{array}{l}\text { malignancy } \ldots \ldots \ldots \ldots \ldots \\
\text { Abortion with dilation and }\end{array}$ & 345 & 56 & 78 & 43 & 81 & 87 & 1.8 & 1.8 & 1.7 & 2.1 & 1.9 & \\
\hline $\begin{array}{l}\text { curettage of uterus } \ldots \ldots \ldots \ldots \\
\text { Bronchitis and asthma, age }\end{array}$ & 325 & 36 & 68 & 41 & 78 & 102 & 1.3 & 1.6 & 1.4 & 1.5 & 1.2 & 1.1 \\
\hline $\begin{array}{l}0-17 \text { years } \ldots \ldots \ldots \ldots \ldots \\
\text { Simple pneumonia and pleurisy, }\end{array}$ & 313 & 59 & 79 & 50 & 70 & 56 & 3.9 & 3.4 & 3.8 & 4.5 & 4.1 & 3.8 \\
\hline $\begin{array}{l}\text { age } 0-17 \text { years. . . } \ldots \ldots \ldots \\
\text { Tonsillectomy and/or } \\
\text { adenoidectomy, age } 0-17\end{array}$ & 279 & 85 & 63 & 48 & 49 & 35 & 4.7 & 4.1 & 4.9 & 5.0 & 5.2 & 4.9 \\
\hline $\begin{array}{l}\text { years } \ldots \ldots \ldots \\
\text { Inguinal and femoral hernia } \\
\text { procedures, age } 18-69 \text { years } \\
\text { without substantial comorbidity }\end{array}$ & 279 & 52 & 55 & 63 & 68 & 41 & 1.8 & 1.6 & 1.8 & 1.9 & 1.7 & 1.9 \\
\hline $\begin{array}{l}\text { and/or complication . ........ } \\
\text { Diabetes, age greater than }\end{array}$ & 271 & 40 & 50 & 45 & 72 & 64 & 4.2 & 4.2 & 3.9 & 4.4 & 4.2 & 4.2 \\
\hline $\begin{array}{l}35 \text { years } \ldots \ldots \ldots \ldots \ldots \ldots \\
\text { Vaginal delivery with } \\
\text { sterilization and/or dilation }\end{array}$ & 259 & 57 & 43 & 53 & 56 & 50 & 8.2 & 6.2 & 7.2 & 8.2 & 9.4 & 9.7 \\
\hline $\begin{array}{l}\text { and curettage of uterus } \ldots \ldots \\
\text { Other factors influencing }\end{array}$ & 247 & 47 & 47 & 41 & 47 & 65 & 3.6 & 3.4 & 3.3 & 3.5 & 3.7 & 3.8 \\
\hline $\begin{array}{l}\text { health status . . . . . . . . . . } \\
\text { Total cholecystectomy with } \\
\text { common bile duct exploration, } \\
\text { age less than } 70 \text { years without } \\
\text { substantial comorbidity and/or }\end{array}$ & 242 & 35 & 38 & 39 & 58 & 73 & 3.5 & 3.1 & 4.0 & 3.5 & 3.6 & 3.4 \\
\hline $\begin{array}{l}\text { complication . . . . . . . . . . . } \\
\text { Bronchitis and asthma, age } \\
18-69 \text { years without } \\
\text { substantial comorbidity and/or }\end{array}$ & 233 & 44 & 41 & 34 & 65 & 48 & 7.8 & 7.2 & 7.5 & 8.4 & 8.4 & 7.4 \\
\hline complication............ & 227 & 60 & 37 & 38 & 45 & 46 & 5.3 & 4.8 & 5.3 & 5.0 & 5.7 & 5.8 \\
\hline
\end{tabular}

consistent for some of the individual DRG's for which average length of stay in small and medium-size hospitals is equal to or greater than the average length of stay in large hospitals (500 or more beds).

The average length of stay associated with a DRG (tables
1-4) allows hospitals to compare their experience with that other hospitals. While comparison is tenuous on a case-by case basis, a hospital with an average length of stay 2,3 , or more days longer than the national average for a specific DRG may need to examine why it is so far from the norm. This kind 


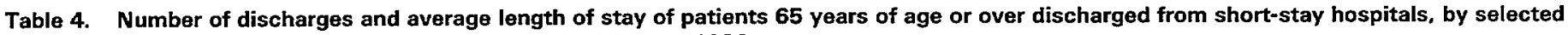
diagnosis-related groups and hospital bed size: United States, 1982

Discharges from non-Federal short-stay hospitals. Excludes newborn infants]

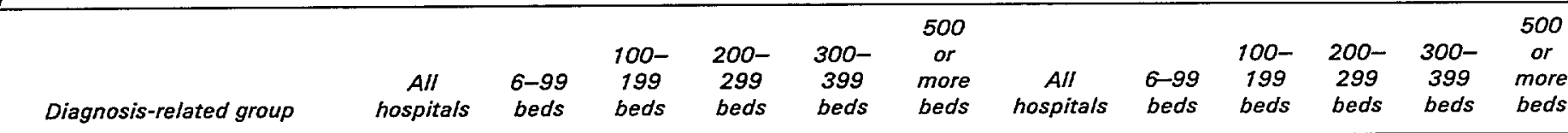

\begin{tabular}{|c|c|c|c|c|c|c|c|c|c|c|c|c|}
\hline \multirow[b]{2}{*}{ All discharges........... } & \multicolumn{6}{|c|}{ Number in thousands } & \multicolumn{6}{|c|}{ Average length of stay in days } \\
\hline & 10,697 & 2,172 & 1,832 & 1,907 & 2,638 & $\overline{2,148}$ & 10.1 & 8.1 & 9.6 & 10.2 & 10.8 & 11.5 \\
\hline $\begin{array}{l}\text { Lens procedures. . . . . . . . . } \\
\text { Atherosclerosis, age greater } \\
\text { than } 69 \text { years and/or } \\
\text { substantial comorbidity and }\end{array}$ & 429 & 32 & 94 & 85 & 118 & 99 & 2.9 & 2.9 & 3.0 & 2.6 & 3.0 & 3.1 \\
\hline complication........... & 427 & 90 & 87 & 80 & 107 & 63 & 8.7 & 7.3 & 8.1 & 8.8 & & 10.2 \\
\hline $\begin{array}{l}\text { Heart failure and shock ....... } \\
\text { Esophagitis, gastroenteritis, and } \\
\text { miscellaneous digestive } \\
\text { disorders, age greater than } \\
69 \text { years and/or substantial } \\
\text { comorbidity and }\end{array}$ & 387 & 113 & 70 & 68 & 82 & 54 & 9.7 & 8.1 & 9.3 & 10.5 & 10.8 & 11.0 \\
\hline $\begin{array}{l}\text { complication. . . . . . . . . . } \\
\text { Chronic obstructive pulmonary }\end{array}$ & 376 & 123 & 60 & 64 & 78 & 51 & 6.7 & 5.6 & 7.2 & 6.9 & 8.3 & 6.5 \\
\hline $\begin{array}{l}\text { disease } \ldots \ldots \ldots \ldots \ldots \ldots \ldots \\
\text { Specific cerebrovascular } \\
\text { disorders except transient }\end{array}$ & 300 & 76 & 48 & 58 & 71 & 48 & 9.8 & 8.1 & 9.5 & 10.7 & 10.6 & 10.5 \\
\hline ischemic attack. . . . . . . . & 294 & 66 & 54 & 60 & 63 & 50 & 15.4 & 11.2 & 15.8 & 16.1 & 14.9 & 20.1 \\
\hline $\begin{array}{l}\text { Simple pneumonia and pleurisy, } \\
\text { age greater than } 69 \text { years } \\
\text { and/or substantial comorbidity }\end{array}$ & & & & & & & & & & & & \\
\hline $\begin{array}{l}\text { and complication } \ldots \ldots \ldots \ldots \\
\text { Unrelated operating room }\end{array}$ & 276 & 98 & 45 & 42 & 56 & 36 & 10.7 & 9.3 & 10.9 & 11.7 & 11.8 & 11.6 \\
\hline $\begin{array}{l}\text { procedures } \ldots \ldots \ldots \ldots \ldots \ldots \\
\text { Diabetes, age greater than }\end{array}$ & 226 & 29 & 42 & 45 & 58 & 52 & 17.3 & 14.1 & 17.3 & 16.0 & 17.5 & 19.9 \\
\hline 35 years $\ldots \ldots \ldots \ldots \ldots$ & 208 & 50 & 37 & 44 & 44 & 33 & 10.0 & 8.2 & 9.2 & 10.3 & 10.9 & 12.1 \\
\hline Angina pectoris. ........... & 195 & 54 & 34 & 40 & 44 & 23 & 6.6 & 5.9 & 6.3 & 6.3 & 7.6 & 7.4 \\
\hline $\begin{array}{l}\text { Medical back problems ....... } \\
\text { Cardiac arrhythmia and } \\
\text { conduction disorders, age } \\
\text { greater than } 69 \text { years and/or } \\
\text { substantial comorbidity and }\end{array}$ & 186 & 45 & 33 & 36 & 41 & 31 & 9.3 & 7.8 & 8.3 & 8.5 & 11.6 & 10.2 \\
\hline $\begin{array}{l}\text { complication } \ldots \ldots \ldots \ldots \ldots \\
\text { Circulaton disorders with acute } \\
\text { myocardial infarction without } \\
\text { cardiovascular complications, }\end{array}$ & 181 & 49 & 31 & 33 & 37 & 30 & 7.1 & 5.7 & 6.6 & 7.8 & 7.8 & 8.5 \\
\hline discharged alive $\ldots \ldots \ldots \ldots$ & 174 & 35 & 27 & 31 & 46 & 35 & 12.2 & 11.2 & 11.6 & 12.1 & 12.9 & 12.9 \\
\hline Hypertension. . . . . . . . . & 158 & 39 & 31 & 29 & 27 & 32 & 7.9 & 6.2 & 7.3 & 9.3 & 7.7 & 9.6 \\
\hline $\begin{array}{l}\text { Transient ischemic attacks .... } \\
\text { Bronchitis and asthma, age } \\
\text { greater than } 69 \text { years and/or } \\
\text { substantial comorbidity and }\end{array}$ & 155 & 36 & 34 & 30 & 32 & 24 & 7.6 & 5.5 & 7.5 & 7.6 & 9.7 & 8.2 \\
\hline $\begin{array}{l}\text { complication . . . . . . . . . . . } \\
\text { Transurethral prostatectomy, } \\
\text { age greater than } 69 \text { years } \\
\text { and/or substantial comorbidity }\end{array}$ & 148 & 47 & 31 & 25 & 28 & 17 & 8.3 & 6.9 & 9.6 & 8.7 & 8.8 & 8.2 \\
\hline $\begin{array}{l}\text { and complication } \ldots \ldots \ldots \ldots \\
\text { Kidney and urinary tract } \\
\text { infections, age greater than } \\
69 \text { years and/or substantial } \\
\text { comorbidity and }\end{array}$ & 144 & 14 & 31 & 27 & 38 & 33 & 9.3 & 9.2 & 8.3 & 10.2 & 9.2 & 9.5 \\
\hline complication $\ldots \ldots \ldots \ldots \ldots$ & 142 & 48 & 26 & 20 & 31 & 18 & 8.5 & 7.2 & 9.2 & 8.5 & 9.2 & 9.4 \\
\hline $\begin{array}{l}\text { Respiratory neoplasms. . . . . . . } \\
\text { Hip and femur procedures } \\
\text { except major joint, age greater } \\
\text { than } 69 \text { years and/or } \\
\text { substantial comorbidity and }\end{array}$ & 137 & 22 & 19 & 19 & 40 & 37 & 10.9 & 7.7 & 10.8 & 10.0 & 11.8 & 12.4 \\
\hline complication ............ & 135 & 16 & 23 & 24 & 40 & 32 & 19.5 & 18.3 & 18.1 & 17.9 & 20.3 & 21.3 \\
\hline
\end{tabular}

of comparison may be worthwhile as a starting point, but even within a DRG, average length of stay is not an exact measure of resource consumption.

The change to prospective payment for Medicare inpatient reimbursement is likely to affect areas such as-cost savings, quality of care, medical records keeping, and certain areas of medical practice. However, for at least two reasons data currently available on DRG's from the NHDS (this report and a 
similar report using 1981 data $^{\text {l) }}$ should not be used to evaluate the success of prospective payment. First, the prospective payment program was not implemented until October 1983, and, second, historical trends must be studied to shed light on shortterm changes in hospital utilization.

For example, from 1981 to 1982 average length of stay decreased 0.4 days for patients 65 years of age and over, and

\section{References}

${ }^{1}$ National Center for Health Statistics, R. Pokras: Diagnosis-related groups using data from the National Hospital Discharge Survey. United States, 1981. Advance Data From Vital and Health Statistics. No. 98. DHHS Pub. No. (PHS) 84-1250. Public Health Service. Hyattsville, Md., July 20, 1984.

${ }^{2}$ National Center for Health Statistics, E. J. Graves: Utilization of short-stay hospitals, United States, 1982 Annual Summary. Vital and Health Statistics. Series 13, No. 78. DHHS Pub. No. (PHS) 841739. Public Health Service. Washington. U.S. Government Printing Office, Aug. 1984.

${ }^{3} \mathrm{~J}$. Alsofrom: Playing the numbers. Medical World News, Oct. 24, 1983, pp. 38-55.

${ }^{4}$ R. B. Fetter, S. Youngsoo, J. L. Freeman, and others: Case mix definition by diagnostic related groups. Medical Care 18(2), Supplement. (Copyright 1980: Used with the permission of Medical Care.)

${ }^{5}$ National Center for Health Statistics, W. F. Lewis: Utilization of short-stay hospitals, summary of nonmedical statistics, United States, 1972. Vital and Health Statistics. Series 13, No. 19. DHEW Pub. No. (HRA) 75-1770. Health Resources Administration. Washington. U.S. Government Printing Office, June 1975.

${ }^{6}$ Natiunal Center for Health Statistics, M. G. Sirken: Utilization of short-stay hospitals, summary of nonmedical statistics, I'nited States,

\section{Technical notes}

\section{Survey methodology}

\section{Source of data}

The National Hospital Discharge Survey (NHDS) encompasses patients discharged from short-stay hospitals, exclusive of military and Veterans Administration hospitals, located in the 50 States and the District of Columbia. Only hospitals with six or more beds and an average length of stay of less than 30 days for all patients are included in the survey. Discharges of newborn infants are excluded from this report.

The universe of the survey consisted of 6,965 short-stay hospitals contained in the 1963 Master Facility Inventory of Hospitals and Institutions. New hospitals were sampled for inclusion in the survey in $1972,1975,1977$, and 1981. The sample for 1982 consisted of 550 hospitals. Of these, 71 refused to participate and 53 were out of scope either because the hospital had gone out of business or because it failed to meet the definition of a short-stay hospital. Thus 426 hospitals partici- some specific DRG's also showed a reduction in average length of stay. However, length of stay in short-stay non-Federal hospitals has been decreasing for over a decade (the average length of stay for patients 65 years of age and over was 12.2 days in $1972^{5}$ compared with 10.1 days in 1982), and it will take more time to understand the effects prospective payment will have, if any, on hospital utilization.
1965. Vital and Health Statistics. Series 13, No. 2. PHS Pub. No. 1000 . Public Health Service. Washington. U.S. Government Printing Office, Aug. 1967.

${ }^{7}$ National Center for Health Statistics, M. J. Witkin: Utilization of short-stay hospitals by characteristics of discharged patients, United States, 1965. Vital and Health Statistics. Series 13, No. 3. PHS Pub. No. 1000. Public Health Service. Washington. U.S. Government Printing Office, Dec. 1967.

${ }^{8}$ National Center for Health Statistics, R. Pokras: Surgical and nonsurgical procedures in short-stay hospitals, United States, 1979. Vital and Health Statistics. Series 13, No. 70. DHHS Pub. No. (PHS) 83-1731. Public Health Service. Washington. U.S. Government Printing Office, Feb. 1983.

${ }^{9}$ U.S. Public Health Service and Health Care Financing Administration: International Classification of Diseases, 9th Revision, Clinical Modification. DHHS Pub. No. (PHS) 80-1260. Public Health Service. Washington. U.S. Government Printing Office, Sept. 1980.

${ }^{10}$ Federal Register. Vol. 48, No. 171, Part II, Rules and Regulations, Sept. 1, 1983.

pated in the survey during 1982 and provided approximately 214,000 abstracts of medical records.

\section{Sample design}

All hospitals with 1,000 or more beds in the universe of short-stay hospitals were selected with certainty in the sample. All hospitals with fewer than 1,000 beds were stratified, the primary strata being 24 size-by-region classes. Within each of these 24 primary strata, the allocation of the hopsitals was made through a controlled selection technique so that hospitals in the sample would be properly distributed with regard to type of ownership and geographic division. Sample hospitals were drawn with probabilities ranging from certainty for the largest hospitals to 1 in $\mathbf{4 0}$ for the smallest hospitals.

Sample discharges were selected within the hospitals using the daily listing sheet of discharges as the sampling frame. These discharges were selected by a random technique, usually on the basis of the terminal digit or digits of the patient's medical 
record number, a number assigned when the patient was admitted to the hospital. The within-hospital sampling ratio for selecting sample discharges varied inversely with the probability of selection of the hospital.

\section{Data collection and estimation}

The sample selection and the transcription of information from the hospital records for abstract forms were performed by the hospital staff or by representatives of the National Center for Health Statistics or by both. The data were abstracted from the face sheets of the medical records. All discharge diagnoses were listed on the abstract in the order of the principal one, or the first-listed one if the principal one was not identified, followed by the order in which all other diagnoses were entered on the face sheet of the medical record.

Statistics produced by NHDS are derived by a complex estimating procedure. The basic unit of estimation is the sample inpatient discharge abstract. The estimating procedure used to produce essentially unbiased national estimates in NHDS has three principal components: inflation by reciprocals of the probabilities of sample selection, adjustment for nonresponse, and ratio adjustment to fixed totals. These components of estimation are described in appendix I of two earlier publications. ${ }^{6,7}$

\section{Diagnosis-related groups}

The diagnosis-related groups (DRG's) used in this report were produced using the most current DRG program available at the time (summer of 1983). This is a computer program that groups patients into DRG's based on diagnostic, surgical, and patient information. The program is maintained and is commercially available at Health Systems International (DRG Support Group, 100 Broadway, New Haven, Conn. 06511). However, the actual program used to produce estimates in this report was obtained from the Health Care Financing Administration.

To help interpret the data in this report, two points are worth mentioning. First, the entire NHDS file was used to produce estimates, including outliers. None of the data was excluded, or trimmed, because of an abnormally long length of stay. Second, the NHDS only codes three ICD-9-CM Class 4 procedures: ${ }^{8,9}$ circumcision, code 64.0 ; episiotomy, code 73.6; and removal of intrauterine contraceptive device, code 97.71 . In certain instances Class 4 procedures can alter the DRG designation for a patient. The effect of not coding these procedures in the NHDS on determining DRG's is unknown, but probably quite small. In all other respects, the DRG's presented in this report are consistent with those in the Federal Register of Thursday, September 1, 1983. ${ }^{10}$

In publications from the National Center for Health Statistics using NHDS data, several schemes have been used to group patients into categories based on either their diagnoses or the procedures performed. These groups were developed to report general purpose statistics to the many users of NHDS data, and any similarity between the titles of those categories and DRG titles is coincidental.

NOTE: $\mathrm{A}$ list of references follows the text.

\section{Sampling errors and rounding of numbers}

The standard error is a measure of the sampling variability that occurs by chance because only a sample, rather than an entire universe, is surveyed. The relative standard error of the estimate is obtained by dividing the standard error by the estimate itself and is expressed as a percent of the estimate. Table I shows relative standard errors for discharges and firstlisted diagnoses for 1982. The standard errors for average lengths of stay are shown in table II. Estimates have been rounded to the nearest thousand. For this reason detailed figures within tables do not always add to the totals.

\section{Tests of significance}

In this report, the determination of statistical inference is based on the two-tailed Bonferroni test for multiple comparisons. Terms relating to differences, such as "higher" and "less," indicate that the differences are statistically significant. Terms such as "similar" or "no difference" mean that no statistically significant difference exists between the estimates being compared. A lack of comment on the difference between any two estimates does not mean that the difference was tested and found to be not significant.

\section{Definition of terms}

Patient-A person who is formally admitted to the inpatient service of a short-stay hospital for observation, care, diagnosis, or treatment. In this report the number of patients refers to the number of discharges during the year, including any multiple discharges of the same individual from one or more short-stay hospitals.

Table I. Approximate relative standard errors of estimated number of discharges and first-listed diagnoses: United States, 1982

\begin{tabular}{|c|c|c|}
\hline & Size of estimate & $\begin{array}{c}\text { Relative } \\
\text { standard } \\
\text { error }\end{array}$ \\
\hline 10,000 & & 16.3 \\
\hline 50,000 & $\ldots$ & 10.2 \\
\hline 100,000 & $\ldots \ldots \ldots \ldots$ & 8.5 \\
\hline $300,000 \ldots$ & $\ldots \ldots \ldots \ldots \ldots \ldots \ldots \ldots \ldots \ldots$ & 6.6 \\
\hline $500,000 \ldots$ & 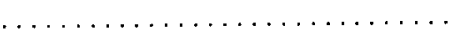 & 5.9 \\
\hline $1,000,000$ & $\ldots \ldots \ldots \ldots$ & 5.1 \\
\hline $4,000,000$ & & 4.0 \\
\hline
\end{tabular}

Table II. Approximate standard errors of average lengths of stay by number of discharges: United States, 1982

\begin{tabular}{|c|c|c|c|c|}
\hline \multirow[b]{2}{*}{ Number of discharges } & \multicolumn{4}{|c|}{ Average length of stay in days } \\
\hline & 2 & 6 & 10 & 20 \\
\hline & \multicolumn{4}{|c|}{ Standard error in days } \\
\hline 10,000 & 0.7 & 1.2 & 1.7 & 2.2 \\
\hline 50.000 & 0.3 & 0.7 & 1.0 & 1.4 \\
\hline 100,000 & 0.3 & 0.6 & 0.9 & 1.2 \\
\hline 500,000 & 0.2 & 0.5 & 0.8 & 0.9 \\
\hline $1,000,000$ & 0.2 & 0.5 & 0.8 & 0.7 \\
\hline $5,000,000$ & 0.2 & 0.5 & 0.8 & $\ldots$ \\
\hline
\end{tabular}


Average length of stay-The total number of patient days accumulated at time of discharge by patients discharged during the year divided by the number of patients discharged.

Age-Patient's age refers to age at birthday prior to admission to the hospital inpatient service.

Discharge-Discharge is the formal release of a patient by a hospital; that is, the termination of a period of hospitalization by death or by disposition to place of residence, nursing home, or another hospital. The terms "discharges" and "patients discharged" are used synonymously.

Geographic region-Hospitals are classified by location in one of the four geographic regions of the United States that correspond to those used by the U.S. Bureau of the Census:

Region

States included

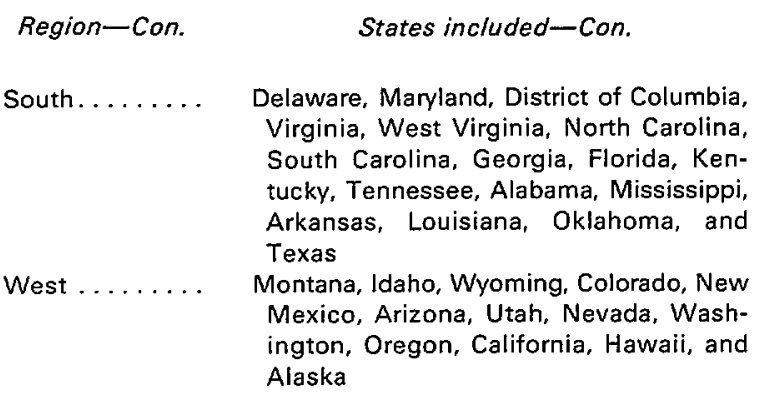

Hospitals-Short-stay special and general hospitals have six beds or more for inpatient use and an average length of stay of less than 30 days. Federal hospitals and hospital units of institutions are not included.

Bed size of hospital-Size is measured by the number of beds, cribs, and pediatric bassinets regularly maintained (set up and staffed for use) for patients; bassinets for newborn infants are not included. In this report the classification of hospitals by bed size is based on the number of beds at or near midyear reported by the hospitals.

\section{Recent Issues of Advance Data From Vital and Health Statistics}

No. 104. Fecundity and Infertility in the United States, 1965-82 (In preparation)

No. 103. Use of Services for Family Planning and Infertility: United States, 1982 (Issued Dec. 20, 1984)

No. 102. Use of Contraception in the United States: 1982 (Issued Dec. 4,1984 )

\section{Suggested Citation}

National Center for Health Statistics, R. Pokras and K. K. Kubishke: Diagnosis-Related Groups Using Data From the National Hospital Discharge Survey, United States, 1982. Advance Data From Vital and Health Statistics. No. 105. DHHS Pub. No. (PHS) 85-1250. Public Health Service. Hyattsville, Md., Jan. 18, 1985.
No. 101. 1983 Summary: National Hospital Discharge Survey (Issued Sept. 28, 1984)

No. 100. CAT Scan Use in Short-Stay Non-Federal Hospitals: United States, 1979-82 (Issued Sept. 28, 1984)
U.S. DEPARTMENT OF HEALTH AND

HUMAN SERVICES

Public Health Service

National Center for Health Statistics

3700 East-West Highway

Hyattsville, Maryland 20782

OFFICIAL BUSINESS

PENALTY FOR PRIVATE USE, $\$ 300$

To receive this publication regularly, contact the National Center for Health Statistics by

calling $301436-8500$

\section{Copyright Information}

The National Center for Health Statistics has obtained permission from the copyright holders to reproduce certain quoted material in this report. Further reproduction of this material is prohibited without specific permission of the copyright holders. All other material contained in the report is in the public domain and may be used and reprinted without special permission; citation as to source, however, is appreciated.
THIRD CLASS MAIL BULK RATE

POSTAGE \& FEES PAID PHS/NCHS

PERMIT No. G-281 